\title{
L'opposition militaire autour des sociétés secrètes dans l'armée
}

Bernard Gainot

\section{(2) OpenEdition \\ 12 Journals}

Édition électronique

URL : https://journals.openedition.org/ahrf/7583

DOI : 10.4000/ahrf.7583

ISSN : 1952-403X

Éditeur :

Armand Colin, Société des études robespierristes

Édition imprimée

Date de publication : 1 décembre 2006

Pagination : 45-58

ISSN : 0003-4436

Référence électronique

Bernard Gainot, «L'opposition militaire autour des sociétés secrètes dans l'armée », Annales historiques de la Révolution française [En ligne], 346 | Octobre/Décembre 2006, mis en ligne le 01 décembre 2009, consulté le 22 avril 2022. URL : http://journals.openedition.org/ahrf/7583; DOI : https://doi.org/ 10.4000/ahrf.7583

Ce document a été généré automatiquement le 22 avril 2022.

Tous droits réservés 


\title{
L'opposition militaire autour des sociétés secrètes dans l'armée
}

\author{
Bernard Gainot
}

1 Le chantier de l'opposition militaire a été très largement visité. La mise au point de Paul Gaffarel dans la revue La Révolution française est reprise dans la plupart des manuels; elle est centrée sur les rivalités personnelles et le ressentiment nourri par certains de ses pairs, notamment Bernadotte et Moreau, envers celui qui a su s'affranchir des règles tacites qui réglaient les rapports entre les seigneurs de la guerre et le pouvoir civil sous le Directoire. Elle évoque encore la très vive hostilité de nombreux officiers envers le Concordat, et non des moindres, comme le général Delmas qui se demande s'il valait bien la peine de faire des dizaines de milliers de morts pour arriver à une telle mascarade. Indépendamment du « complot des libelles » à l'armée de l'Ouest, où il y eut bien « conspiration militaire " sans la moindre tentative d'exécution, les pratiques oppositionnelles se limitent à des propos privés, rapportés par des dénonciateurs. Comme le dit l'auteur : «Il n'y eut donc pas à vrai dire d'opposition, mais seulement des velléités d'opposition militaire contre Bonaparte $»^{1}$.

2 Par ailleurs, les paroles séditieuses sont généralement circonscrites au cercle des officiers supérieurs. En nous en tenant aux sources d'archives, nous n'avons que très peu d'échos des opinions véritables de simples soldats. Gaffarel mentionne bien cette mutinerie du 1er régiment d'artillerie à Turin, événement effectivement aussi important qu'exceptionnel, mais les raisons du mécontentement restent obscures et nous ne pouvons, sans étude plus approfondie, considérer cette mutinerie comme une manifestation d'opposition. Il nous est tout aussi difficile de savoir ce que pensait vraiment la troupe lorsque ses officiers apportaient une adhésion unanime au Consulat à vie et au rétablissement du pouvoir héréditaire que lorsque le commandant militaire du département de la Charente, Malet, refusait de faire pavoiser à Angoulême pour saluer l'établissement de l'Empire. Lorsqu'on établit le parallèle avec l'effervescence des premières années de la Révolution, avec les témoignages individuels de militaires lors du vote sur la Constitution de 1793, on mesure bien le succès de la reprise en mains de l'institution par l'encadrement hiérarchique, tel que l'a étudié Jean-Paul Bertaud ${ }^{2}$. 
3 Pourtant, l'évocation du développement des sociétés secrètes dans l'armée par Charles Nodier renvoie précisément à cette période de la fin du Directoire et du Consulat. J'ai essayé, dans d'autres articles, de montrer comment, à travers une stratégie d'écriture qui relève tant du rituel du secret, que de l'opportunité contextuelle, le récit de Nodier pouvait être considéré comme témoignage de l'état de l'opposition à l'Empire au printemps $1814^{3}$. Il reste à évaluer si ce témoignage a valeur rétrospective, comme le prétend Nodier, pour toute la période 1795-1814. Cette valeur est refusée par la plupart des historiens, parce qu'ils n'y voient que « construction romanesque ». Ce n'était pas $\mathrm{du}$ tout le cas des historiens du XIX ${ }^{\mathrm{e}}$ siècle, dont Gaffarel. Il n'hésite pas à prendre comme source les renseignements fournis par Nodier: «Une société secrète s'était formée dans l'armée, dont les membres prêtaient serment de rester fidèles aux institutions républicaines. Ils se nommaient les Philadelphes. On connaît mal les dispositions et les résolutions des adhérents, car ils gardaient le secret le plus absolu, et, quand ils étaient surpris, égaraient la police par de fausses révélations. Charles Nodier, le premier historien des Philadelphes, a, comme à plaisir, embrouillé le récit de leurs entreprises, à tel point qu'on a pu l'accuser d'avoir inventé de toutes pièces et comme forgé un roman de conspiration militaire. Les Philadelphes ont pourtant existé, et, à diverses reprises, ils ont prouvé leur existence par des actes sérieux $»^{4}$.

Bien entendu, notre propos ne sera pas ici de revenir sur ce procès. Remarquons simplement qu'à travers l'action d'un officier comme Oudet, Nodier a voulu mettre en lumière celle d'officiers, qui ne sont certes pas de rang subalterne, mais non pourvus d'un commandement d'armée, comme Bernadotte, Moreau, Brune ou Lannes. Gaffarel en cite d'autres: Fournier, Boccechiampe, Guibal, et Argoud, qui sera le personnage central de cette communication. "Citons parmi les officiers que le Premier Consul poursuivit de sa haine un certain Argout ou d'Argoult, général divisionnaire en retraite, qui s'était fait remarquer par l'exagération de ses plaintes et de ses attaques contre le gouvernement. Il fut arrêté et interné à l'île d'oléron ; mais il s'échappait de temps à autre et allait à La Rochelle et à Rochefort où il cherchait à nouer des intelligences avec les officiers de ces deux places. Ses allées et venues, ses imprudences, les conciliabules qu'il essayait d'organiser, tout fut bientôt découvert. Dénoncé par un capitaine dont il avait espéré faire un complice, Argout fut transporté à la Guyane où il ne tarda pas à périr victime du climat $»^{5}$.

5 Retournons donc aux sources pour développer l'affaire du général Pierre Argoud, puis jalonner quelques pistes pour aborder une étude plus globale de l'opposition militaire.

6 L'affaire de la «machine infernale », en décembre 1800, avait fourni le prétexte à la déportation de 130 personnalités de l'opposition démocratique, considérées comme " anarchistes", dont un certain nombre de militaires. Plusieurs d'entre eux sont assignés à résidence sur l'île d'oléron, ou à Belle-Île, en attendant une destination plus lointaine ${ }^{6}$. Le cas du général de brigade Pierre Argoud a retenu plus particulièrement notre attention.

7 Pierre Argoud est né à Saint-Geoire, dans l'Isère, le 26 juin 1763. Son père était un « bourgeois » de la ville, mais il va évoluer différemment. Il entre très jeune au service, en 1777, au Corps Royal de la Marine à Toulon. Il a fait la guerre d'Amérique, en 1782. Il reprend du service au bataillon des volontaires de l'Ain, en 1791, comme capitaine. Il a été nommé général de brigade, à titre provisoire, en brumaire an II (novembre 1793), par les représentants du peuple Saint-Just et Lebas, en mission à l'armée du Rhin. Ce grade est confirmé le 15 fructidor an III (août 1795) seulement, après avoir été contesté, 
comme bien d'autres, au printemps 1795. Les circonstances de cette nomination, un avancement jugé trop rapide, l'ont rendu suspect à Aubry, chargé de la réforme des états-majors. Alors, le général Pichegru intervient en sa faveur, soulignant ses grandes qualités militaires, et son utilité au service. Il est réformé le 29 pluviôse an VI (17 février 1798), par suite de ses nombreuses blessures. Il habite à cette date à Strasbourg, 45 , faubourg de Saverne, et il vit avec celle qui se présente par la suite comme « son épouse ", Marie-Anne Lenatzen.

En fait, Pierre Argoud a épousé auparavant, en 1791, Marie Ponsard, dont il a eu trois enfants. L'épouse délaissée se manifeste de nouveau en avril 1801, se plaignant d'être sans nouvelles du père de sa progéniture, dont elle sait par ailleurs qu'il «a épousé plusieurs autres femmes aux armées, sans avoir divorcé $»^{7}$. De tels cas de bigamie, voire de polygamie, n'étaient pas rares dans les armées de cette époque. Entre autres, j'ai trouvé deux cas semblables dans les dossiers de généraux oppositionnels : la troisième épouse de Pierre Quantin se plaint, dans une lettre à l'empereur datée de 1808, d'avoir été abandonnée ${ }^{8}$. Quantin, qui fut régulateur de la Société Politique de Marseille en 1799, est très mal noté, fauteur de malversations, repéré pour son manque de loyalisme envers le régime. Le général Sarrazin, auteur de Mémoires à la réputation douteuse, est pareillement en butte à des poursuites pour « bigamie ». L'intérêt porté à une situation qui est loin d'être exceptionnelle dans les armées de cette époque, mais qui est plus systématiquement relevée pour des officiers dont l'inspection militaire soupçonne le loyalisme envers le régime, interroge précisément sur la représentation de la société militaire construite par le dit régime. Nous y reviendrons.

9 Pierre Argoud est un officier oppositionnel, depuis la période du Directoire. C'est à cette époque que le général aurait évolué vers les démocrates, par mécontentement à l'égard du Directoire, ce qui est une évolution classique chez les nombreux officiers écartés de l'armée par suite des compressions d'effectifs'. Le 21 thermidor an VII (8 août 1799), il écrit au représentant Pierre-Joseph Briot, du Conseil des Cinq-Cents, chef de file du néo-jacobinisme parlementaire, pour lui demander d'appuyer sa remise en activité. Celle-ci lui est accordée, puis différée en vendémiaire an VIII (septembre 1799). Il est employé au Conseil de révision des conscrits, à Dijon, une voie de garage. Il tient par ailleurs un cabaret à Saint-Florentin, ville voisine d'Auxerre, où il a de solides relations dans les milieux néo-jacobins ${ }^{10}$. Ses " discours exaltés ", les aspects troubles de sa vie privée, attirent sur lui l'attention de la police, et il est arrêté le 1er thermidor an X (21 juillet 1802), sur intervention du premier inspecteur de la gendarmerie nationale, le général Moncey ${ }^{11}$.

Nouveau déménagement: Argoud fixe sa résidence à Oléron, mais il fait des tournées sur le continent, en trompant la surveillance des autorités locales, à Saintes, à Rochefort, à La Rochelle. Le maire d'oléron, Boudin, l'a fait enfermer dans une des salles de la maison commune à son retour. "J'ai acquis la certitude que cet individu, pendant la tournée qu'il a faite dans le continent, y a tenu les propos les plus séditieux contre le gouvernement, particulièrement à Rochefort, où il a dit qu'il se préparait un second mouvement contre le Premier Consul, qu'il pouvait compter sur 12000 hommes pour le moment, ainsi que sur les hommes de couleur de l'île, que tous les déportés faisaient cause commune, que le préfet de ce département, le sous-préfet de Marennes, le maire du Château, le commandant de l'île, le commissaire de police de Bordeaux (le citoyen Pierre Pierre) étaient de la conspiration [...]». 
11 Une femme est arrivée avec Argoud : elle est également considérée comme dangereuse. Elle s'appelle Edmée James, c'est la femme du citoyen Joseph Lefebvre de Dijon, un employé aux fournitures militaires, mais Argoud la présente comme son épouse : c'est devenu une habitude... «Il serait décent de renvoyer cette troisième épouse chez sa mère ", estime le maire. Par ailleurs, Argoud est isolé dans l'île, sauf de l'ex-général Sabathier, «son digne compagnon qui lui a prêté son chapeau de général et son brevet pour faire ses courses dans le continent ». Sabathier est un autre général néo-jacobin, activiste qui aurait tenté d'organiser une opposition militaire lors du coup d'État de Brumaire $^{12}$.

L'̂̂le d'oléron est alors, à l'instar d'autres îles, un territoire spécial, quasiment placé en dehors de l'empire de la Constitution, selon la formule des décrets du régime consulaire. C'est à la fois un centre pénitentiaire, un lieu de résidence surveillée pour un certain nombre d'opposants jacobins, un dépôt colonial, où sont reléguées plusieurs compagnies d'hommes de couleur, suspectes par nature, pourrait-on dire à l'heure où les colonies de la Guadeloupe et de Saint-Domingue sont en pleine insurrection contre les corps expéditionnaires de Leclerc et Richepanse, venus désarmer les troupes noires pour mieux restaurer l'ancien régime colonial ${ }^{13}$. La cohabitation n'est guère facile entre cet agitateur, et les autres prisonniers de l'île d'oléron, qui aimeraient bien se faire oublier. Le commandant d'armes de l'île, et son adjudant de place, unissent leurs plaintes à celles des déportés Louis et Vatar (l'ancien rédacteur du Journal des hommes libres) : l'ex-général tient des propos extrêmement séditieux contre le gouvernement. Il a compromis tous les déportés en annonçant publiquement qu'ils étaient mêlés à un vaste mouvement en préparation contre le Premier Consul, et qu'il pouvait aussi compter sur les hommes de couleur en garnison dans l'île. Les autorités, dans la situation spécifique du territoire insulaire, ont la hantise d'une conjonction des oppositions. Les mêmes Louis et Vatar tiennent de l'ex-général Sabathier lui-même qu'il a prêté à Argoud son chapeau de général et son brevet pour faire sa tournée. Sabathier est tous les jours avec les hommes de couleur, dans les cabarets et les cafés ${ }^{14}$.

Pourquoi Argoud a-t-il échappé à la surveillance des autorités pour se rendre discrètement sur le continent ? L'enquête menée sur la tournée continentale d'Argoud a pour informateur principal un personnage anonyme, qui est en réalité un mouchard de la police secrète, Dumesnil, chef de bataillon de la 5e demi-brigade d'artillerie de marine de Rochefort, organisateur des compagnies d'hommes de couleur sur l'île d'Oléron ${ }^{15}$. Argoud s'est présenté chez lui, à Rochefort, le 9 vendémiaire, comme général divisionnaire, portant trois étoiles au chapeau et des brevets délivrés par le Directoire exécutif. Ils sortent sur la place d'armes, puis Argoud propose d'aller déjeuner à son hôtel, Le cheval blanc, faubourg de La Rochelle. Là, Argoud tient le discours suivant: le gouvernement est injuste, mais il espére bientôt renverser la machine. C'est lui qui est à la tête de tous ces projets. «Il vient de faire une tournée de douze jours, au cours de laquelle il a rencontré beaucoup d'amis, dont plusieurs chefs de corps qui sont prêts à le seconder ; il a vu aussi beaucoup de sous-officiers. À ceux-ci, il fait valoir qu'il n'y a plus d'espoirs d'avancement, et il promet à la troupe 15 sols par jour, la ration, et habillés comme ils l'étaient au commencement de la Révolution ; qu'il avait déjà gagné la compagnie des noirs qui est à lîle d'oléron, parce qu'il leur a fait apercevoir que l'intention du gouvernement est de les renvoyer aux Isles pour les rendre comme autrefois. Il dit encore qu'il a réuni une somme de 600000 francs pour son projet, qu'en faisant mouvement sur Rochefort, il s'emparerait de la Caisse 
publique. Il affirme son intention de supprimer les droits des barrières, ainsi que l'impôt sur les portes et fenêtres, de chasser les prêtres réfractaires et les nobles ». Ce projet anticipe sur les multiples plans échafaudés par la suite par d'autres conspirateurs comme Malet. Toutefois, le rôle central dévolu à la compagnie des hommes de couleur, selon l'un de leurs instructeurs, dans un projet de conspiration, alors que la suspicion à leur égard n'a jamais été aussi forte de la part des autorités consulaires, ne manque pas de nous interroger sur une manipulation. La conversation avec l'informateur se prolonge en promenade et en étapes dans deux débits de boisson, le café Petiteau, et un petit limonadier, chez qui Argoud réitère ses propos. Puis il affirme son intention de rentrer à La Rochelle, où il a rendez-vous avec des officiers de la garnison. Cette dernière affirmation est certainement placée pour garantir le sérieux du projet dans l'esprit de son interlocuteur.

Le lendemain soir, Argoud se présente de nouveau chez X... (Dumesnil), qui le reçoit le lendemain matin. X... a fait prévenir un de ses camarades (Beneaut ou Legat), devant lequel Argoud répète tous ses propos. Comme le camarade lui demande les raisons de sa destitution, il réplique que c'est parce qu'il a prêté son appartement pour une réunion de patriotes. S'il voulait écrire au Premier Consul, avec lequel il est très lié, il serait immédiatement réintégré. Mais il ne veut pas servir un tel monstre, un déserteur qui a abandonné l'armée d'Égypte après avoir fait empoisonner 8000 malades dans un hôpital, « que le premier médecin ayant refusé, il l'avait ordonné à un adjoint qui avait exécuté l'ordre». Il a fait assassiner Kléber et Desaix, et Argoud peut fournir les preuves, qui seraient imprimées un jour. Cette allusion à l'affaire des pestiférés de Jaffa, et plus généralement aux péripéties de l'expédition d'Égypte, est déjà partie intégrante de la «légende noire » de Bonaparte, véhiculée sur une large échelle par les Anglais, mais aussi dans les nombreux libelles qui sont colportés clandestinement par les militaires et les démocrates ${ }^{16}$. Il faut voir à ce sujet le texte violemment antibonapartiste qui circulait sous le manteau après le 18 Brumaire, Dialogue entre le Turc et le Démocrate, dont l'auteur, Bernard Metge, avait été fusillé au début de l'année 1801. Le fait de la retrouver dans ce dossier d'accusation peut constituer un indice délibérément placé par la police pour suggérer les liens entre les nombreuses oppositions, interne et externe, au Consulat.

Le sous-préfet de Marennes invite à plus de retenue en vendémiaire an XI (septembre 1802) ; il relativise les plans de conspiration soi-disant révélés par le maire et la police secrète: "Ce maire met beaucoup d'importance aux propos extravagants de l'exgénéral Argout $[s i c]$; je pense que nos administrés (au moins la partie sensée) nous rendront assez de justice pour croire que nous ne partageons nullement les sentiments d'un homme qui s'annonce l'ennemi d'un gouvernement que notre inclination particulière comme citoyens, et notre devoir comme fonctionnaires publics nous rendent aussi cher $\aleph^{17}$. Pour éviter à l'avenir les déplacements d'Argoud, il va demander aux préposés des douanes de surveiller plus attentivement les passeports. Le souspréfet confirme qu'Argoud s'est rendu à La Rochelle, à Saintes, à Saint-Jean d'Angély, et même à Angoulême, pour revenir à La Rochelle. Là, les gendarmes de la brigade de La Rochelle l'ont arrêté. Ils donnent son signalement: il a 39 ans, les cheveux et les sourcils noirs, les yeux gris, une barbe noire. Mis en cause, Sabathier écrit une lettre au préfet pour nier tout en bloc ; il n'a pas contribué à l'évasion d'Argoud, il n'a pas prêté son chapeau et ses brevets. Argoud a ses propres brevets. Quant aux accusateurs, ce sont des "hommes méchants, connus pour leurs sottises; Vatar et Louis qui étaient aussi à Rochefort, sans permission ", leurs camarades, «à qui aucun gouvernement ne 
convient que le leur ", Méhée et Daubigny. Sabathier cherche à couvrir Argoud; si ce dernier est parti sur le continent, c'était pour y chercher des subsistances, du pain et de la viande, pour lui, pour Sabathier et son fils, en pension sur l'île d'oléron.

Le sous-préfet de l'arrondissement de Saint-Jean d'Angély donne également des renseignements sur la visite qu'Argoud fit à Saint-Jean, où il est passé le 10 vendémiaire. Le récit est certainement plus révélateur que celui du mouchard sur les motivations véritables de l'ex-général. Il venait de Saintes où il avait fait dire à Terray, chef d'escadron du 20e régiment de dragons en garnison dans la ville, qu'ayant été appelé à la préfecture, mais devant passer par Saint-Jean, il fit tenir à sa disposition un dragon avec un cheval. Terray était son ancien aide de camp à l'armée du Rhin. Il vint en personne au devant d'Argoud. Un repas est préparé avec le chef de brigade commandant le 20e dragons. Il ne se tint aucun propos contre le gouvernement. La soirée se prolongea au Café de la Comédie, où on ne se livra qu'à une conversation gaie et galante. En somme, l'aigreur du général viendrait surtout de la rage d'avoir perdu, avec son emploi, la position sociale et les mille et un petits avantages y afférant. Un tempérament bouillant, et cette aigreur peuvent se traduire en propos vite considérés comme "séditieux " par une police omniprésente et zélée. D’autant plus que le temps de la libre parole, bien mal distinguée de la "gasconnade » si répandue dans les cercles militaires, n'est pas si loin. Lorsque l'on respecte les égards auxquels il estime avoir droit, lorsque finalement, il est en confiance avec ses relations, l'ex-général ne ressemble guère au conspirateur imprudent. C'est un gaillard, certainement vantard, surtout soucieux de détailler ses bonnes fortunes féminines à ses camarades.

17 Le 8 brumaire an XI (30 octobre 1802), le sous-préfet de Marennes précise qu'Argoud et Sabathier ont été enfermés à la citadelle. Quant à la compagnie des hommes de couleur, elle est sortie la veille de l'île, pour se rendre en Italie. Le maire Boudin n'est pas tranquille pour autant. Le 17 nivôse an XI (7 janvier 1803), il eut la surprise de trouver Argoud en ville, se promenant dans les cabarets. Il envoie aussitôt deux gendarmes pour l'arrêter chez le cabaretier Bourguignon, mais Argoud leur oppose l'autorisation du commandant de la citadelle à sortir tous les trois jours. Le commandant lui a donné un garde national pour planton, mais ils se sont enivrés tous les deux, et ont cherché querelle à plusieurs personnes du village, en les traitant d'esclaves, et en proférant des menaces contre le maire ( il ferait venir le boudin en douille »). Et le maire s'indigne, témoignant ainsi de l'antipathie personnelle qu'il éprouve envers l'officier: "Voilà donc ce prétendu chef de parti, ce général couvert de blessures et plein d'honneur [...] souffleté dans les cabarets et menant la vie la plus crapuleuse ; voilà les cadeaux qu'on destine à l'île d'oléron, je vous avouerait [sic] que cela seul me répugne à continuer les fonctions de maire ». Boudin a fait couper les vivres à Argoud, car celui-ci prétendait ne pas vouloir les payer, arguant que c'est son droit de jouir de son traitement de réforme.

18 J'arrête ici cette étude de cas, parce que nous avons suffisamment d'éléments pour réfléchir à la façon dont se forge une "conspiration » à cette époque. Les principaux intéressés avaient coutume de dénigrer la tendance constante de la police secrète à produire des conspirations - ce qui est la justification même des émoluments des préposés de ladite police - sous le nom de conspiration des œufs rouges. Faudrait-il alors s'en tenir à des affaires d'inimitié personnelle, à l'animadversion entre le militaire bravache et gaillard, et le notable bourgeois de province qui craint pour ses biens, femme et filles comprises, et pour son autorité ? Ce serait alors une variante locale du sempiternel conflit entre les autorités civiles et les autorités militaires. 
19 Le général réformé est un personnage aigri et mécontent; les racines profondes de ce mécontentement, toutefois, tiennent à la perte d'une situation acquise, et non à une opposition politique ferme, toujours supposée, jamais clairement établie, au vu des sources, par une pratique avérée et incontestable (l'association, la proclamation écrite, le geste irrémédiable). Il y aurait bien mécontentement, il n'y aurait pas opposition. En fait, il y a un processus de marginalisation de l'officier écarté du service actif, processus au terme duquel il n'est plus maître de son image, telle qu'elle se forge à travers les pièces constitutives de son dossier, où s'accumulent les témoignages à charge. Apparaît alors en filigrane un contre-modèle de l'officier tel que le souhaite le régime bonapartiste, un individu policé par le monde des notables, respectable et respecté. Ces témoignages récurrents de bigamie, d'endettement suspect ou crapuleux, de négligence dans la tenue ou le comportement, n'atteignent que des individus que le régime marginalise pour des raisons autres que les faits qui leur sont reprochés. Prenons un autre exemple individuel, qui avait également retenu mon attention, pour des raisons identiques de dévalorisation de la figure sociale de l'opposant. Le général Pierre-Jean Treich-Desfarges fut un élément actif du mouvement néo-jacobin en Corrèze, très proche du général Antoine Marbot. Réformé en janvier 1800, il est de retour dans son bourg de Meymac où, exerçant conjointement les professions de notaire et de médecinaccoucheur, propriétaire d'une des plus grosses maisons du bourg, il pourrait parfaitement être défini comme notable, si on ne retenait que le revenu et le talent comme critères exclusifs de définition. Il y a un autre critère essentiel qui est la réputation, la fama, un artefact produit par les rapports de police. Le capitainecommandant la gendarmerie du département de la Corrèze en 1808 décrit l'ex-général, allant « dans les foires avec son habit de général, un bonnet sous son chapeau et des sabots aux pieds, se mettant à table dans les cabarets avec le premier paysan qui veut bien lui payer à boire ». On le présente comme n'ayant point de société, hors les paysans. Il conseille ces derniers, leur donne des avis « comme s'il était avocat ${ }^{18}{ }^{2}$. C'est donc bien la figure de l'anti-notable qui ressort de ce dossier. Mais, après tout, il n'est que de lire les mémoires des officiers les plus représentatifs de cette époque pour savoir que la multiplicité des liaisons féminines, ou les dettes de jeu, ou les rixes, ou les beuveries canailles en compagnie du tout-venant, sont pratique courante dans les états-majors. Pourtant, on ne retient des traces de celles-ci que dans les dossiers des officiers que le régime a écartés, pour des raisons qui n'ont rien à voir parfois, ni avec un comportement effectif, ni même avec des opinions politiques précises.

Le dossier, comme toute source d'archives, est donc inséré dans de complexes stratégies d'écriture et de composition. Élargissons un peu la perspective, en formulant quelques hypothèses, tant sur la nature de l'opposition, que sur celle du régime qu'elle prétend combattre. Il faut se garder de ramener un peu hâtivement l'affaire du général Argoud à l'anecdote, négligeant les nombreux indices politiques dont elle est émaillée. L'évolution du Consulat (la signature du Concordat, la large amnistie accordée aux émigrés, le renforcement du pouvoir personnel de Bonaparte avec le Consulat à vie) mécontente un grand nombre d'officiers républicains, dont plusieurs se retrouvent dans les départements de l'Ouest, à l'état-major de Bernadotte ${ }^{19}$. Ce que redoute particulièrement le gouvernement, c'est une conjonction des oppositions. Il craint que sa politique de "restauration coloniale", en rupture si manifeste avec les valeurs républicaines, n'alimente le mécontentement général, évident au sein de nombreux cercles militaires, mais présent également dans l'opinion. Yves Benot a relevé de nombreuses traces de ce mécontentement à travers les bulletins de police, et 
notamment l'exploitation qui en est faite par les «exclusifs $»^{20}$ (les jacobins). Ces craintes durcissent en retour les mesures discriminatoires et la répression contre les personnes de couleur. Certes, l'opposition jacobine dirige surtout sa propagande vers les milieux militaires pour les dissuader de partir aux Antilles.

Le contexte de l'année 1802 est bien spécifique. Contrairement à un cliché largement répandu, et forgé a posteriori, le régime consulaire ne s'est pas installé dans le consensus. Les incertitudes sur les orientations politiques du nouveau régime, son manque évident de légitimité, entretiennent un climat de rumeurs, d'insatisfactions latentes et de défis verbaux. À en croire le général Puget-Barbantane, qui vient de traverser la France en fructidor an IX (juillet 1801), «j'allai voir Fouché, alors ministre de la police générale, et je lui dis que sur la route on ne parlait que de conspirations pour assassiner Bonaparte ${ }^{21} »$. Il est vrai que le général, ami du peintre Topino-Lebrun, ne porte pas plus le Consulat que le Directoire dans son cœur. À propos du 18 Brumaire, il note : «La République n'était plus; le Directoire disparut comme une ombre, et avec lui périt la liberté, dont il avait préparé la ruine par un aveuglement qui n'a pas d'exemple». Cet aveuglement tient au fait d'avoir voulu offrir «le spectacle d'un gouvernement qui voulait une république sans républicains $»^{22}$. La Paix d'Amiens, en 1802, va fournir l'occasion d'épurer les cadres. Une opinion, largement répandue, veut que l'expédition de Saint-Domingue ait été l'un des moyens de se débarrasser des éléments militaires les plus rétifs au nouvel ordre consulaire. Dans une analyse très fouillée de la composition du corps expéditionnaire, un travail universitaire a cherché à vérifier l'hypothèse avancée par la plupart des historiens d'Haïti, et par le colonel Malenfant, partisan déclaré de Moreau et adversaire de l'expédition, selon laquelle Bonaparte aurait délibérément sacrifié les soldats qui avaient servi sous son adversaire. Rien de concluant : sur vingt demi-brigades d'infanterie de ligne, dix ont fait partie de l'armée du Rhin, les autres ont appartenu à des corps d'armée très divers ${ }^{23}$, dont la 79e qui a participé au coup d'État de Brumaire. Certes, s'il note que l'expédition a permis "d'envoyer quelques officiers turbulents se racheter une conduite ${ }^{24}$, le simple bon sens oblige à constater que nul ne pouvait prévoir au départ l'hécatombe finale qui a emporté l'aventure coloniale. Mais, toutefois, si la dissidence n'avait pas été limitée à un corps d'armée spécifique, mais avait affecté l'ensemble de l'institution militaire?

Nous voici donc ramenés au silence et aux lacunes des sources. Défions-nous méthodiquement des Mémoires d'officiers, écrits dans un tout autre contexte, avec une perspective toute différente de ce qu'elle était sous le Consulat. Un inventaire systématique de ces Mémoires militaires reste à faire, dans l'optique de ce qui a été réalisé pour les Mémoires de la Terreur. Par défaut, nous voilà réduits à glaner quelques éléments chez certains officiers marginalisés. C'est ce que nous avons fait avec PugetBarbantane. Nous disposons encore des Mémoires du général Sarrazin, écrits dans une perspective de réhabilitation sous la Restauration, puisque leur auteur était passé chez les Anglais en 1810. Sarrazin se présente alors comme légitimiste, mais comment faire l'économie de l'époque à laquelle il écrit, alors qu'une lecture attentive, et débarrassée de tout préjugé hostile, permet de voir en Sarrazin un opposant de longue date, attaché à l'état-major de Bernadotte, intimement lié à des officiers proches des néo-jacobins, comme le général Bonnamy, qui fut un proche de Championnet?

23 Tout dépend de la spécificité et de la qualification d'une opposition militaire républicaine. Ce qui repose la question, et du césarisme, et de l'analyse du régime issu du 18 Brumaire. Écoutons ce que disent les contemporains, et d'abord le texte de Fontanes, 
souvent attribué à Lucien Bonaparte, parce que le ministre de l'Intérieur a couvert sa diffusion : Parallèle entre César, Cromwell, Monck et Bonaparte. Ce texte donne une version du régime, quelque peu différente des interprétations courantes. Il s'efforce de démarquer Bonaparte de la figure historique de César ; "mais César, en accablant le parti le plus juste, et Bonaparte, en ralliant les Citoyens contre le parti des brigands ; et ici, Bonaparte et César, qui se ressemblent comme guerriers, différent comme politiques [...] $»^{25}$. César a soulevé les fureurs de la multitude contre la «sagesse patricienne ", qui constitue le vrai rempart de la Liberté. "Brutus fut le défenseur de l'Ordre social contre l'Anarchie, de la Propriété contre la Loi agraire, du Peuple contre la populace. Robespierre et ses partisans, qui se réclament de Brutus, ont tout confondu. C'est contre les démagogues que Brutus s'est armé ; César a été le chef des démagogues ». Bonaparte a rallié la classe des propriétaires et des gens instruits contre la multitude ${ }^{26}$. Si on suit bien le texte, Bonaparte fut le rempart des notables contre une alliance des démagogues, des "anarchistes » (terme alors couramment employé pour désigner les néo-jacobins) et des militaires. "Si la tyrannie des assemblées vous épouvante, quel sera votre refuge, si ce n'est la puissance militaire? » La "puissance militaire ", ce sont quelques chefs qui se combattent sans cesse, et que leur faiblesse rend cruels. Néron, Caligula, Claude, remplacent César assassinée ${ }^{27}$. Laissons donc de côté l'appel à l'instauration d'un pouvoir héréditaire, anticipation du Consulat à vie auquel on tend généralement à réduire ce texte, pour y voir un appel au ralliement des notables contre la menace d'un pouvoir militaire niveleur, contre lequel le Directoire épuré de ses éléments les plus conservateurs, ne présentait plus de garanties suffisantes. Fantasmes et manipulation de l'opinion publique? Peut-être pas, si l'on tient compte de l'image que pouvait offrir alors une armée, certes animée d'un fort ressentiment envers une société civile dans laquelle elle ne veut voir que des " pékins " qui la méprisent, mais aussi conservatoire de valeurs égalitaires et " populistes », pour aller vite, qui incarnent un retour à la République de l'an II, généralement brandi comme un épouvantail dans les élites dirigeantes. Dans l'un des textes qui reflètent le mieux la sensibilité néo-jacobine de l'époque, le pamphlet de François Dubreuil, largement diffusé en forme d'adresse à Bonaparte, l'auteur ne craint pas de reprendre le parallèle avec Cromwell pour stigmatiser l'illégitimité du régime issu de Brumaire : "Là, nouveau Cromwell, tenant un fouet à la main, tu parais dans le Sénat pour lui annoncer que la Constitution, acceptée par le Peuple et les armées, va être remplacée par un triumvirat ${ }^{28}$ ».

Bien entendu, le fossé entre ces opinions, en tant que telles, et les indices manifestes d'une opposition militaire organisée, est béant. Pour le combler, il faudrait analyser les composantes d'une culture nationale-populaire fortement imprégnée de jacobinisme et de revanche sociale, qui ne serait pas réductible au " militarisme ", réduction à laquelle se prêtent hâtivement nombre d'études récentes. Il faudrait encore avoir des témoignages probants de l'existence de structures clandestines que Charles Nodier, encore lui, affirme avoir existé dès l'époque du Directoire. Nous avons bien des indices de réseaux, mais ces réseaux sont-ils des réunions de clientèles, ou bien des structures organisées autour d'un projet politique, dont on mesure mal en quoi il pourrait offrir une alternative au pouvoir des notables tel que la République Directoriale, et bien plus encore le Consulat l'organisent? La recherche historique bute sur la positivité de la source écrite, réduite aux innombrables témoignages policiers (polices et contrepolices s'enchevêtrent depuis l'an IV), aux rares confidences, souvent bien postérieures, des activistes, comme celle que livre le correspondant de Didier en 1807 : 
«Il existait des réunions de patriotes, une maçonnerie où tout se tramait sans écrit, à la jésuite, qu'il en faisait partie, qu'il y avait des ramifications étendues [...] $»^{29}$.

\section{NOTES}

1.Paul GAFFAREL, «L'opposition militaire sous le Consulat », La Révolution française, 12/1887, p. 1100.

2.Jean-Paul BERTAUD, La Révolution armée. Les soldats-citoyens et la Révolution française, Paris, Robert Laffont, 1979, IIIe partie, p. 267-334.

3.Bernard GAINOT, « Pratiques politiques et stratégies narratives. Hypothèses de recherche sur les conspirations militaires : la "conspiration Malet" de 1808 » dans Politix, Économie politique du secret, 54/2001, p. 95-117. Et du même : « Réflexions sur une forme politique de transition. À propos de la conspiration Malet de 1808 » dans La plume et le sabre. Hommages offerts à Jean-Paul Bertaud, Paris, Publications de la Sorbonne, 2002, p. 513-524.

4.Paul GAFFAREL, « L'opposition militaire... », op. cit., p. 995- 996.

5.Ibid., p. 992-993. Les sources utilisées par l'auteur sont essentiellement la notice biographique, bien informée par ailleurs, de Jean DESTREM, Les déportations du Consulat et de l'Empire, Paris, 1885, p. 311.

6.AD Charente-Maritime, 4M6 (1), Correspondance concernant les déportations dans les îles. 7.Service historique de la Défense, dossier « Pierre Argoud », g.b. 649 (2).

8.Service historique de la Défense, dossier « Pierre Quantin », g.b. 285 (2).

9.AN, AF III 187-197, Travail du ministère sur les officiers, ans IV-VIII. Une pièce de son dossier, datée de nivôse an VI (janvier 1798), donne pour adresse « chez le citoyen Pélegrin, 1010, rue Dominique, au Gros Caillou ». Ce bourg, à proximité de l'Hôtel des Invalides, est alors un centre d'agitation militaire et politique d'importance. Argoud se déplace dans la capitale pour obtenir sa réintégration.

10.Idem, p. 278-285.

11.Service historique de la Défense, dossier g.b. 649 (2), et AD Charente-Maritime, 4M6 (1).

12.Sabathier a été déporté à cette époque, par arrêté du 20 brumaire an VIII, et non à la suite de la «machine infernale ». Voir Jean DESTREM, Les déportations du Consulat et de l'Empire, op. cit.

13.Frédéric RÉGENT, « Origines, desseins et destins des rebelles » dans 1802 en Guadeloupe et à Saint-Domingue : réalités et mémoire, $\mathrm{n}^{\circ}$ spécial de la Société d'histoire de la Guadeloupe, 2003, p. 31-60.

14.AD Charente-Maritime, Procès-verbal de Boudin, maire d'Oléron, en date du 18 vendémiaire de l'an XI (10 octobre 1802).

15.Le rapport de Dumesnil est adressé au préfet maritime de La Rochelle, Martin. 16.Bernard GAINOT, «Un itinéraire démocratique post-thermidorien : Bernard Metge », dans Pour la Révolution française, recueil d'études réunies par Christine LE BozEC et Éric WAUTERS, Rouen, Publications de l'Université de Rouen, 1998, p. 93-106. 
17.AD Charente-Maritime, 4 M 6 (1). Rapport du sous-préfet de Marennes, 18 vendémiaire an XI (10 octobre 1802).

18.Bernard GAINOT, « Le général Treich-Desfarges. Pratiques politiques en Corrèze sous la Révolution ", dans Actes du $117^{e}$ Congrès du CTHS, Clermont-Ferrand, 1992, p. 145-159. 19.Édouard Guillon, Les complots militaires sous le Consulat et l'Empire, Paris, Plon, 1895. Paul GAFFAREL, op. cit., p. 865, 982, et 1096.

20.Yves BENOT, La démence coloniale sous Napoléon, Paris, La Découverte, 2006, p. 95.

21.Mémoires du lieutenant-général Puget-Barbentane, publiés par lui-même, Paris, Pichon-

Béchet, 1827, p. 218.

22.Idem, p. 216.

23. Mayeul MACE, Novembre 1803 : fin de campagne. La défaite du corps expéditionnaire français à Saint-Domingue, mémoire de maîtrise sous le direction de Bernard Gainot, Université Paris1-Sorbonne, 2002, p. 37 et annexe III.

24.Idem, p. 41.

25.Parallèle entre César, Cromwell, Monck et Bonaparte, BNF, Lb (43) 215, p. 10.

26.Idem, p. 11.

27.Idem, p. 14.

28.François DUBReUIL, Dubreuil à Bonaparte, BNF, Lb (43) 31, 1er frimaire an VIII, p. 12. Les termes sont soulignés par nous.

29.Témoignage cité par Raymonde MONNIER, Répertoire du personnel sectionnaire parisien en l'an II, Paris, Publications de la Sorbonne, 1985, p. 36.

\section{RÉSUMÉS}

L'histoire de l'opposition militaire rencontre l'obstacle majeur de la fiabilité des sources. La rareté des sources directes amène à s'en remettre aux archives policières, ou à des reconstructions littéraires, comme celle de Charles Nodier. Aussi nombre d'historiens préfèrentils ne voir là que " roman conspiratif », ce qui, à notre avis, est une facilité d'écriture valorisant à l'excès la positivité du témoignage direct.

Pour rouvrir le chantier, nous proposons de partir d'études de cas individuels et, pour ce faire, de prendre ici en exemple le séjour du général Argoud à l'île d'Oléron en 1802. En croisant les rapports des autorités administratives, les sources policières, le dossier personnel du général, et ceux des autres relégués militaires de la période sur l'île, nous obtenons le portrait d'un antinotable, dont les traits se dessinent en négatif par rapport à l'image sociale que le régime bonapartiste souhaite modeler pour l'officier supérieur.

Ce portrait, que l'on peut rapprocher de celui d'autres opposants militaires, soulève toutefois d'autres questions, et d'abord celle de la nature du régime à laquelle se trouve confrontée cette opposition.

Military opposition: Secret Societies in the Army. The reliability of sources presents a major obstacle to the history of military opposition. The paucity of direct sources necessitates the use of police archives, or literary reconstructions like that of Charles Nodier. But only a number of historians acknowledge the existence of a «conspiracy novel»-a «fact» that accords too much importance to direct testimony. 
To reopen this field of research, I propose using individual case studies-here, the example of the sejour of general Argoud on the island of Oleron in 1802. By comparing the reports of administrative authorities, police sources, the personal dossier of the General, with those of other military figures of the period on the island, one can trace the portrait of an anti-notable, whose traits are negative compared with the social image that the Bonapartist regime tried to mould of the superior officer. This portrait that can be compared with that of other military opponents of the regime raises other questions, most notably that of the nature of the regime being opposed.

INDEX

Mots-clés : césarisme, opposition, militaires de couleur, notables, conspirations

\section{AUTEUR}

BERNARD GAINOT

Institut d'Histoire de la Révolution française, (Paris I - Sorbonne), 17 rue de la Sorbonne, 75231 Paris cedex 05, bgainot@wanadoo.fr 